\title{
The spin-Dicke effect in OLED magnetoresistance
}

\author{
D. P. Waters ${ }^{1}$, G. Joshi', M. Kavand ${ }^{1}$, M. E. Limes ${ }^{1}$, H. Malissa', P. L. Burn², J. M. Lupton ${ }^{1,3 \star}$ \\ and C. Boehme ${ }^{1 \star}$
}

Pairs of charge-carrier spins in organic semiconductors constitute four-level systems that can be driven electromagnetically'. Given appropriate conditions for ultrastrong coupling ${ }^{2}$-weak local hyperfine fields $B_{\text {hyp }}$, large magnetic resonant driving fields $B_{1}$ and low static fields $B_{0}$ that define Zeeman splittingthe spin-Dicke effect, a collective transition of spin states, has been predicted ${ }^{3}$. This parameter range is challenging to probe by electron paramagnetic resonance spectroscopy because thermal magnetic polarization is negligible. It is accessed through spin-dependent conductivity that is controlled by electron-hole pairs of singlet and triplet spin-permutation symmetry without the need of thermal spin polarization ${ }^{4}$. Signatures of collective behaviour of carrier spins are revealed in the steady-state magnetoresistance of organic light-emitting diodes (OLEDs), rather than through radiative transitions. For intermediate $B_{1}$, the a.c.-Zeeman effect appears. For large $B_{1}$, a collective spin-ensemble state arises, inverting the current change under resonance and removing power broadening, thereby offering a unique window to ambient macroscopic quantum coherence.

Macroscopic phase coherence is a hallmark of many exotic states of matter such as superconductivity, ferromagnetism or BoseEinstein condensation. Such coherence may also emerge between two-level systems, where it is mediated by electromagnetic fields, as described by the Dicke effect in collisional narrowing ${ }^{5}$ and superradiance ${ }^{6}$. Collective behaviour may already arise within a pair of interacting two-level systems ${ }^{7}$, an observation that can potentially be extended to the prototypical two-level system of an electron spin. For pairs of charge-carrier spins in organic semiconductors, with driving fields $B_{1}$ exceeding the hydrogen-induced random local hyperfine field ${ }^{1} B_{\text {hyp }}$ and approaching the magnitude of the static magnetic field $B_{0}$, a collective macroscopic spin phase has been predicted to emerge ${ }^{3}$. Under these conditions, when the spin-Rabi splitting becomes comparable to the Zeeman splitting, the electromagnetic field links individually resonant spin pairs together, forming a spin-Dicke state analogous to that in the dipolar Dicke effect ${ }^{5-7}$. These macroscopic effects are observable through measurements of electronic recombination rates, which depend on spin-permutation symmetry of the pair ${ }^{8}$.

We monitor the electron-hole recombination current in an OLED, where positive and negative charges are injected into a thin film of an organic semiconductor from opposite electrodes. As the charges drift through the material, they can capture each other on intermolecular length scales owing to weak dielectric screening. These weakly coupled intermolecular electron-hole pairs ${ }^{9}$ can ultimately recombine on individual molecules to form a molecular excited state, or exciton, which gives rise to electroluminescence. The subsequent discussion focuses on carrier pairs and not on excitons, which have spin $S=0$ or 1 . Because the carriers possess spin $1 / 2$, there are four quantum-mechanical substates of the electron-hole pair. Incoherent mixing of these states occurs on timescales of the spin-lattice interaction, $T_{1}$, provided a relaxation mechanism exists ${ }^{10,11}$. More importantly, the spins precess coherently in local magnetic fields, in particular around nuclear magnetic moments, leading to coherent spin-state mixing on timescales of the phase coherence, $T_{2}$ (ref. 1). At room temperature, charge-carrier spins in the OLED material poly[2-methoxy-5-(2'ethylhexyloxy)-1,4-phenylenevinylene] (MEH-PPV) are characterized by long spin coherence and relaxation times, $T_{2} \approx 350 \mathrm{~ns}$ and $T_{1} \approx 40 \mu \mathrm{s}$, respectively ${ }^{1}$. These parameters ensure that even tiny static magnetic fields (weaker than nuclear hyperfine fields) modify spin precession of localized carriers and alter spin-permutation symmetry of the pair, which controls the yields of electron-hole recombination and dissociation ${ }^{1,10-13}$. OLEDs therefore exhibit lowfield magnetoresistance $e^{14-16}$ owing to spatial variations in the local magnetic field experienced by the pairs precessing around hydrogen nuclear magnetic moments.

Figure 1 contrasts $B_{0}$-sweeps of the steady-state OLED current at room temperature with and without an oscillating radiofrequency (RF) field of strength $B_{1}$, with a sketch of the set-up in Fig. 1a. We compare two MEH-PPV derivatives ${ }^{17}$ with hydrogenated and deuterated side groups. For the data obtained from hydrogenated MEH-PPV (Fig. 1b), a small initial dip occurs close to the origin, followed by a large increase. The dip has been assigned to the influence of zero-field splitting within the pair ${ }^{18}$. It is thought to be related to the competition between exciton formation and spin mixing ${ }^{19}$, but is of no further relevance here. We note that the absolute sign of the magnetoresistance, which is determined by the balance of spin-dependent pair recombination and dissociation rates ${ }^{20}$, is also insignificant for the subsequent discussion, because the spin-Dicke effect is manifested in relative changes of steady-state conductivity and its qualitative dependence on the driving field $B_{1}$ (ref. 3). As $B_{0}$ increases to $6 \mathrm{mT}$, spin precession in the hyperfine field is modified, changing spin statistics and thus pair dissociation and recombination rates ${ }^{9,10,21}$. This effect is reversed by an $85 \mathrm{MHz}$ RF field, resulting in a magnetic resonance around $3.05 \mathrm{mT}$, in agreement with the known MEH-PPV $g$-factor of 2.002 (refs 1,9). On-resonance, triplets and singlets are interconverted ${ }^{9,10,22,23}$, compensating the underlying static magnetoresistive effect. The difference between $B_{0}$-sweeps with and without RF radiation gives the characteristic magnetic-resonance lineshape in Fig. 1d, which corresponds to a hyperfine-broadened double-Gaussian function associated with the individual resonances of electrons and holes ${ }^{17}$ (see Supplementary Fig. 1 for discussion).

The influence of $B_{\text {hyp }}$ on magnetoresistance can be tested by deuterating the 2-ethylhexyl side chains of MEH-PPV, shown in

\footnotetext{
${ }^{1}$ Department of Physics and Astronomy, University of Utah, 115 South 1400 East, Salt Lake City, Utah 84112, USA. ${ }^{2}$ Centre for Organic Photonics \& Electronics, School of Chemistry \& Molecular Biosciences, The University of Queensland, Queensland 4072, Australia. ${ }^{3}$ Institut für Experimentelle und Angewandte Physik, Universität Regensburg, D-93040 Regensburg, Germany. `e-mail: john.lupton@ur.de; boehme@physics.utah.edu
} 
a
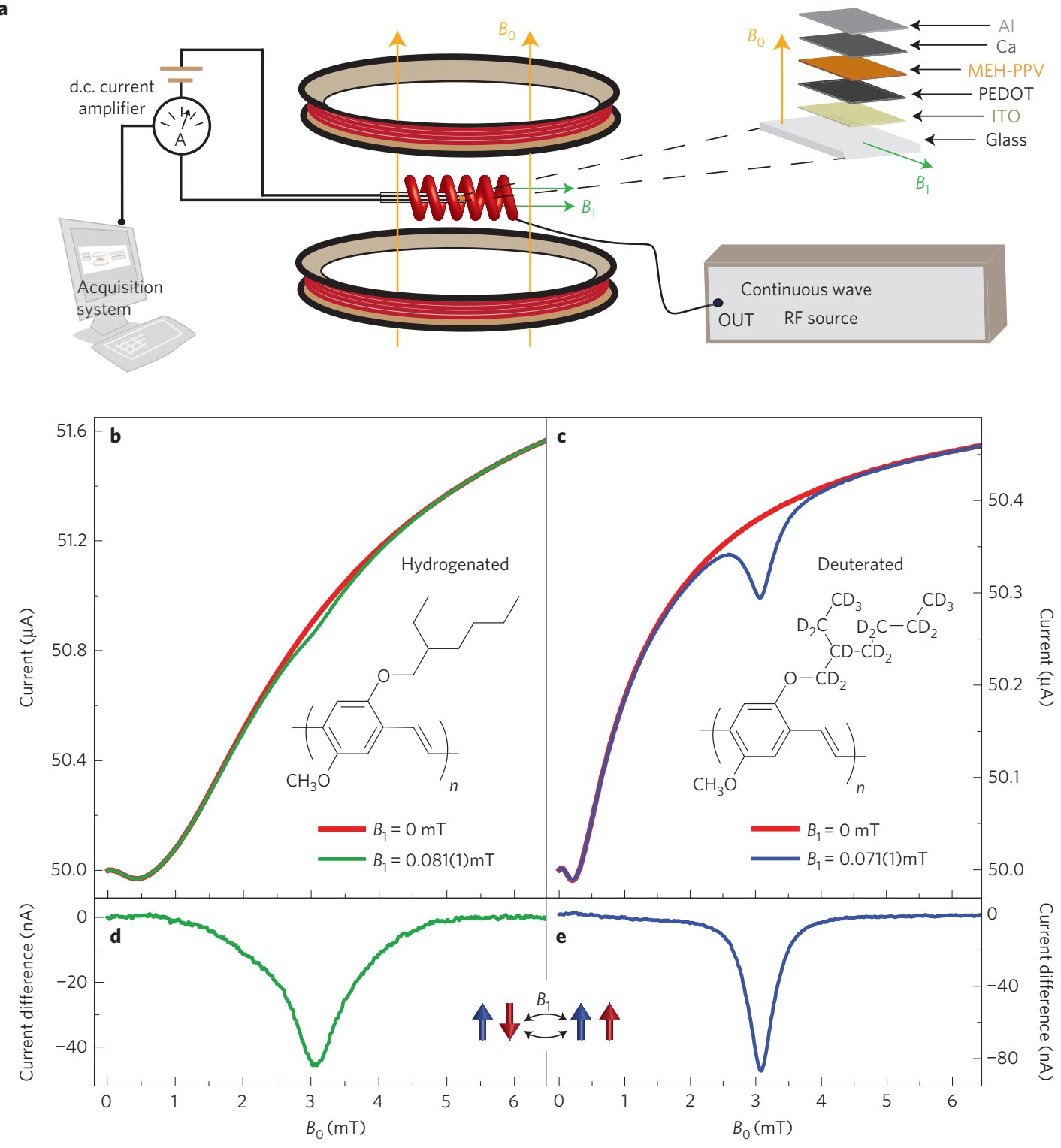

Figure 1 | Magnetoresistance and magnetic resonance in the steady-state current of an OLED at room temperature. $\mathbf{a}$, The OLED is fabricated on a thin narrow strip of glass and contacted with ITO and thin metallic strips to minimize inductive coupling. It is biased using a battery to measure the direct current. A set of Helmholtz coils provides the static $B_{0}$ field, orthogonal to which the oscillating $B_{1}$ field is applied by an RF coil driven by an RF c.w. power source. b,c, Magnetoresistance of MEH-PPV devices (at a bias adjusted to establish a $50 \mu \mathrm{A}$ current) without (red) and with (green or blue) the RF field applied at $85 \mathrm{MHz}$, for samples with hydrogenated (b) and deuterated (c) side chains. d,e, Differential magnetocurrent with and without the RF field, for samples with hydrogenated (d) and deuterated (e) side chains, yielding an electrically detected magnetic resonance in the steady-state current. The cartoon illustrates the electron-hole pair resonance transition between singlet and triplet manifolds. For simplicity, only one of the triplet states is considered in the discussion, but the description applies equally to all triplet states.

Fig. 1c,e, with the magnetoresistance curve appearing steeper for the latter where local hyperfine fields are weaker ${ }^{18,21}$. Under RF irradiation, the dip obtained from deuterated MEH-PPV in Fig. 1c is more pronounced than in Fig. 1b because the hyperfine-broadened resonance narrows ${ }^{18,21}$. Parallels to this RF effect exist in solution-based reaction-yield-detected magnetic resonance of pair processes ${ }^{24-26}$, with the crucial difference being that the OLED current reveals absolute population changes, allowing steady-state detection in magnetoresistance. This signal amplitude enables time-resolved excitation and detection to uncover spin-Rabi oscillations and spin beating due to correlated precession of electrons and holes ${ }^{27}$. To demonstrate that steady-state magnetoresistance arises from coherent spin precession, we pulsed the RF coil and measured the current change as a function of pulse length (Supplementary Fig. 2). The
Rabi frequency increases as the square root of the RF power ${ }^{27,28}$, allowing direct calibration of the resonant driving field $B_{1}$.

Probing magnetic resonance directly in a steady-state current allows for the exploration of interactions between carrier spins and their field-mediated equilibration, which controls magnetoresistance. We distinguish three regimes of interaction between the relevant magnetic fields $B_{0}, B_{\text {hyp }}$ and $B_{1}$ in Fig. $2 \mathrm{a}-\mathrm{c}$. For $B_{1} \ll B_{\text {hyp }}<B_{0}$, the domain of conventional magnetic resonance ${ }^{8}$, only one spin of the electron-hole pair is in resonance with the driving field ${ }^{27}$. For ensembles of weakly coupled pairs, this means that singlet pairs flip to triplets and vice versa. Although spin resonance controls spinpermutation symmetry, which determines conductivity through spin-dependent transitions ${ }^{28}$, the net spin polarization remains zero. For $B_{1} \sim B_{\text {hyp }}$, local magnetic disorder from hyperfine interactions 
a
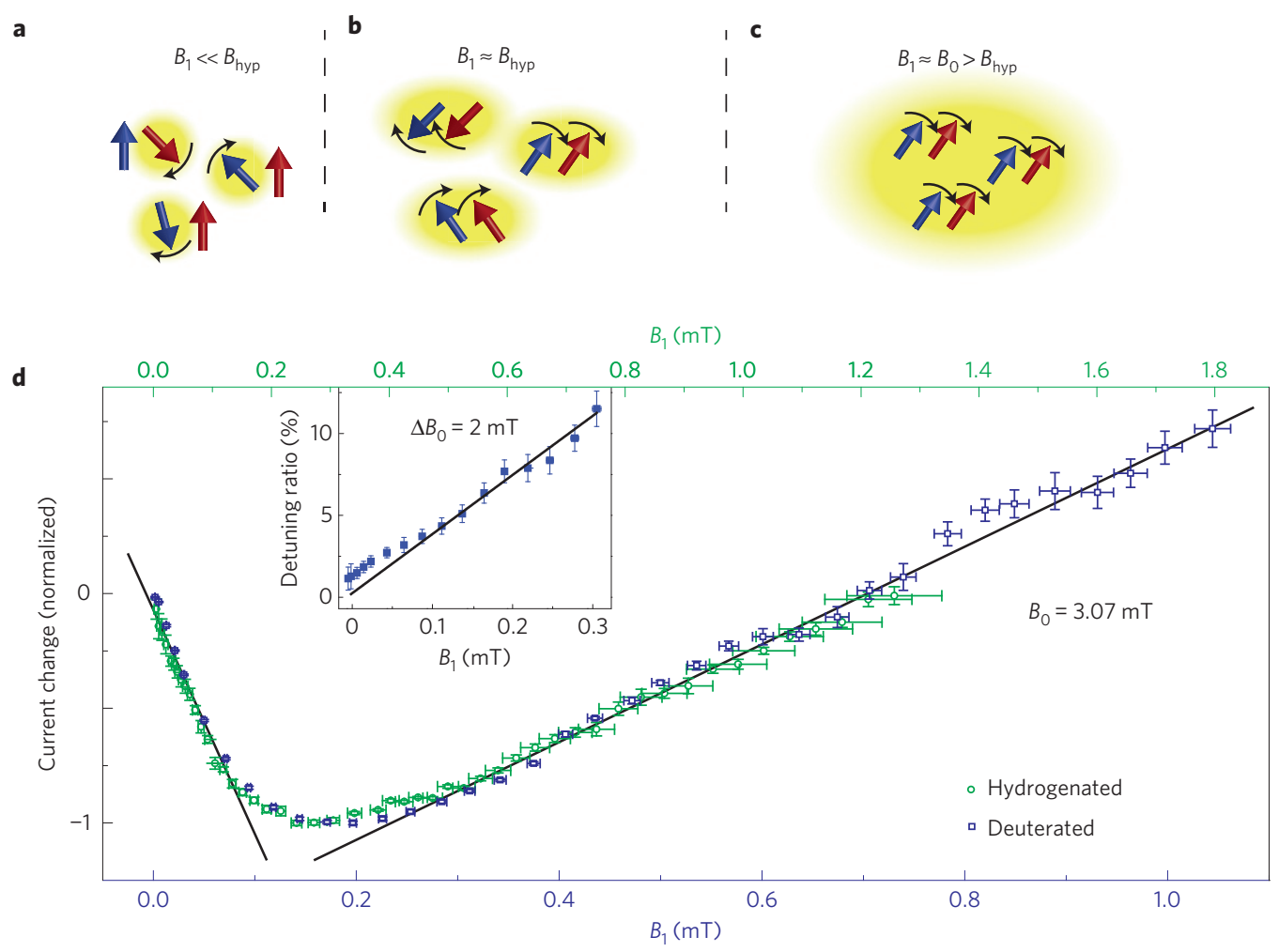

e



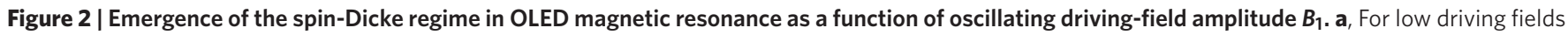
weaker than local hyperfine fields, only an electron or a hole within a carrier pair is excited resonantly. $\mathbf{b}$, As $B_{1}$ increases to exceed $B_{\text {hyp }}$, both electron and hole within the pair become resonant: the pair partners precess in a defined phase. With increasing $B_{1}$, this spin beating gradually cancels out the effect that individual spin-dependent transitions have on conductivity, reducing the current change $\Delta /$. c, For very large driving fields, on the order of the static field $B_{0}$, the spins across the ensemble form a collective state within the RF field, a manifestation of the predicted Dicke effect. For simplicity, only one triplet subensemble is sketched. $\mathbf{d}$, Normalized $\Delta /$ as a function of $B_{1}$, exhibiting the three regimes described, for hydrogenated and deuterated samples. Following ref. 3 , the curves are described by two linear functions (see Supplementary Information for details). Inset: effect of detuning $\Delta B_{0}=2 \mathrm{~m} T$ off-resonance, with the detuning ratio of $\Delta /$ between on- and off-resonance plotted against $B_{1}$ for the deuterated sample. $A$ linear dependence on $B_{1}$ is observed, as predicted in ref. 5. e,f. Plots of $\Delta /$ as a function of $B_{0}$ and $B_{1}$ for hydrogenated (e) and deuterated (f) samples. Both samples show power broadening and a splitting of the resonance due to the a.c.-Zeeman effect, the latter being more clearly resolved under weaker hyperfine coupling. The

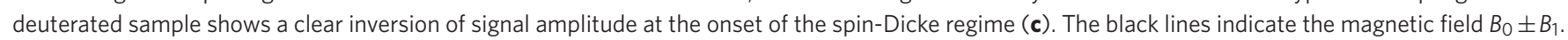
The errors in $\mathbf{d}$ and its inset derive from errors in the current measurements, which are based on estimators for unbiased sample variances obtained as described in the Supplementary Information. 
is overcome so that both the electron and the hole resonate in phase ${ }^{27}$. However, there is still no connection between the individual carrier pairs, which have random phases with respect to the driving field. In the ultrastrong-coupling regime ${ }^{2}$, where $B_{1} \approx B_{0}$, the spin-Dicke effect sets $\mathrm{in}^{3}$, which is equivalent in form to the familiar optical Dicke effect ${ }^{5-7}$. The driving field now defines the Bloch sphere axis, voiding the rotating-wave approximation ${ }^{26}$. In the Dicke regime, spins act together in phase with the driving field, leading to signatures of collective behaviour. However, instead of detecting the coherence directly through the superradiant field from the spin transitions, which is masked by the strong driving field, the collective behaviour of strongly driven spin pairs is revealed in a change of conductivity ${ }^{3}$. As outlined by Roundy and Raikh ${ }^{3}$, the resonance coupling results in new eigenstates of the pair system: a smaller subset of collective pure-singlet character and a dominant triplet-state set. As the pairs become phase-locked by the driving field, a reversal of the resonance sign occurs, enhancing magnetoresistance $^{3}$. This sign reversal, which must coincide with a change in the resonance spectrum, underlines the crucial difference between conventional spin beating ${ }^{27}$ (for $B_{1} \sim B_{\text {hyp }}$ ) and the Dicke effect (when $B_{1} \approx B_{0}$; ref. 3).

The theoretical formalism of OLED magnetoresistance under resonant a.c.-drive ${ }^{3}$ allows several predictions to be made, which we test here. First, current changes as a function of $B_{1}$ should scale with the hyperfine field strength ${ }^{3}$. We therefore plot measurements for hydrogenated and deuterated samples on different linear scales of $B_{1}$ in Fig. 2d, with data for the deuterated (blue) and hydrogenated MEH-PPV (green) giving optimal overlap for a scaling ratio of $1.72(4)$ of the two axes. This number is in good agreement with the ratio of the expectation values for the random hyperfine fields experienced by the charge carriers in the two materials (1.88(13), as obtained from the resonance lineshapes ${ }^{17}$, see Supplementary Information). Qualitatively, theory predicts that when power broadening becomes significant, the magnitude of the resonantly induced current change $\Delta I$ must scale linearly with driving-field amplitude $B_{1}$, rather than quadratically, as would be expected for electromagnetic absorption. This linear rise of $\Delta I$ with $B_{1}$ is shown in Fig. 2 d. $\Delta I$ should saturate, followed by a linear decrease. The slope of the decrease should be smaller than that of the initial increase ${ }^{3}$, as is observed (and discussed in detail in the Supplementary Information). A further test ${ }^{3}$ comes from the effect of $B_{0}$-detuning on $\Delta I$. Off-resonance, $\Delta I$ should be zero, but, as $B_{1}$ increases, the change of resonance lineshape due to power broadening must lead to a quadratic rise in $\Delta I$ with $B_{1}$ for small $B_{1}$ below saturation ${ }^{3}$. We consider in the inset of Fig. $2 \mathrm{~d}$ the detuning ratio $\Delta I_{\mathrm{d}} / \Delta I_{\mathrm{r}}$ as a function of $B_{1} . \Delta I_{\mathrm{r}}$ is defined as the on-resonance current change and $\Delta I_{\mathrm{d}}$ as the detuned current change measured off-resonance, at a field $B_{0}=1.05 \mathrm{mT}\left(\Delta B_{0}=2 \mathrm{mT}\right.$ lower than the on-resonance magnetic field). The detuning ratio normalizes $\Delta I_{\mathrm{d}}$ to the on-resonance current change $\Delta I_{\mathrm{r}}$. As $\Delta I_{\mathrm{r}} \sim B_{1}$ and $\Delta I_{\mathrm{d}} \sim B_{1}^{2}$, $\Delta I_{\mathrm{d}} / \Delta I_{\mathrm{r}}$ is predicted ${ }^{3}$ to be proportional to $B_{1}$. This proportionality is confirmed in the inset of Fig. $2 \mathrm{~d}$, with the deviation close to the origin resulting from hyperfine broadening of the resonance. This broadening breaks one of the conditions set by theory ${ }^{3}$ that the detuning $\Delta B_{0}$ be large $\left(\Delta B_{0} \gg B_{\text {hyp }}\right.$; ref. 3$)$. We conclude that theory ${ }^{3}$ withstands experimental scrutiny and note that the observations differ fundamentally from conventional spin-locking invoked to describe the inversion of reaction-yield-detected RF resonances ${ }^{26}$, because our resonance lineshape depends on $B_{1}$.

Given the experimental tests of theory at low to intermediate $B_{1}$ driving fields ${ }^{3}$, we probed the model for high $B_{1}$ approaching $B_{0}$. At a critical $B_{1}$, the resonances should vanish entirely ${ }^{3}$, beyond which the Dicke regime emerges, manifested by a $\Delta I$ sign reversal and a collapse of the resonance lineshape due to the formation of new eigenstates. Resonance lines of the two devices are plotted versus $B_{1}$ in Fig. 2e,f. For the hydrogenated material, the dominant effect is power broadening, which decreases in strength as fundamental and harmonic spin precession ${ }^{27}$ (that is, spin beating) cancel out their mutual impact on $\Delta I$ for large $B_{1}$. Subsequently, the resonance splits, and then vanishes at approximately $B_{1}=1.1 \mathrm{mT}$. $B_{1}$ is limited by constraints on the RF amplifier and coil design (see Methods). For the deuterated compound, the critical field $B_{1}>B_{\text {hyp }}$ is almost a factor of two lower and the resonance is narrowed, so that the underlying structure of the resonance spectrum is more clearly resolved. The spectrum in Fig. $2 \mathrm{f}$ shows the same power broadening as in Fig. 2e, but also exhibits clear bifurcation above $B_{1} \approx 0.4 \mathrm{mT}>B_{\text {hyp }}$. This splitting is a consequence of the a.c.-Zeeman effect, which is analogous to the Bloch-Siegert shift induced on each spin of the pair by the oscillating field. The expected Zeeman splitting induced by $B_{1}$ on the scale of $B_{0}$ can be deduced from half of the power broadening line width, which is indicated by the black lines in Fig. 2e,f. At higher fields, the resonance suddenly inverts, coinciding with spectral narrowing. Above $B_{1} \approx 0.7 \mathrm{mT}$, most electron-hole spin pairs precess in phase with the driving field and hence with each other, inverting the effect of spin precession on spin-dependent transport (and hence the sign of $\Delta I$ ): new dominant triplet eigenstates emerge for $B_{1} \rightarrow \infty$ (ref. 3 ).

The agreement between model and experiment provides conclusive evidence for the observation of the spin-Dicke effect in OLED magnetoresistance 3 . Although formally analogous to the conventional optical Dicke effect ${ }^{5-7}$, important differences exist in phenomenology, as discussed in the Supplementary Information. Crucially, the spin-Dicke effect is manifested in conductivity, which is controlled by spin-permutation symmetry of the individual pair ${ }^{3}$. It therefore does not scale with ensemble size as superradiance does ${ }^{6}$. Finally, one may speculate that the spin-Dicke effect as revealed in Fig. $2 \mathrm{f}$ could emerge spontaneously in a device, without an external oscillatory field, given sufficiently small $B_{\text {hyp }}$ and large spatiotemporal fluctuations in local magnetic field strength that would constitute an effective $B_{1}$. Such fluctuations could arise from spatial variations in spin-spin couplings experienced by a migrating charge, and should be particularly strong if transport is anisotropic, as in molecular wires ${ }^{29}$. The ability to form collective states of radicalpair spins may find application in coherent control of chemical reactions, but also offers an alternative room-temperature route to permutation-symmetry-based concepts for electrically addressable quantum information processing ${ }^{30}$ and fast resonance-based magnetometry ${ }^{4}$, because the effect of power broadening is reduced.

\section{Methods}

Methods and any associated references are available in the online version of the paper.

Received 11 May 2015; accepted 24 July 2015;

published online 7 September 2015

\section{References}

1. Malissa, H. et al. Room-temperature coupling between electrical current and nuclear spins in OLEDs. Science 345, 1487-1490 (2014)

2. Guenter, G. et al. Sub-cycle switch-on of ultrastrong light-matter interaction. Nature 458, 178-181 (2009).

3. Roundy, R. C. \& Raikh, M. E. Organic magnetoresistance under resonant ac drive. Phys. Rev. B 88, 125206 (2013).

4. Baker, W. J. et al. Robust absolute magnetometry with organic thin-film devices. Nature Commun. 3, 898 (2012).

5. Dicke, R. H. Coherence in spontaneous radiation processes. Phys. Rev. 93, 99-110 (1954)

6. Gross, M. \& Haroche, S. Super-radiance-An essay on the theory of collective spontaneous emission. Phys. Rep. 93, 301-396 (1982).

7. DeVoe, R. G. \& Brewer, R. G. Observation of superradiant and subradiant spontaneous emission of two trapped ions. Phys. Rev. Lett. 76, 2049-2052 (1996).

8. Shinar, J. Optically detected magnetic resonance studies of luminescence-quenching processes in pi-conjugated materials and organic light-emitting devices. Laser Phot. Rev. 6, 767-786 (2012). 
9. McCamey, D. R., Lee, S. Y., Paik, S. Y., Lupton, J. M. \& Boehme, C. Spin-dependent dynamics of polaron pairs in organic semiconductors. Phys. Rev. B 82, 125206 (2010).

10. Steiner, U. E. \& Ulrich, T. Magnetic-field effects in chemical-kinetics and related phenomena. Chem. Rev. 89, 51-147 (1989).

11. Reufer, M. et al. Spin-conserving carrier recombination in conjugated polymers. Nature Mater. 4, 340-345 (2005).

12. Schulten, K., Staerk, H., Weller, A., Werner, H. J. \& Nickel, B. Magnetic-field dependence of geminate recombination of radical ion-pairs in polar-solvents. Z. Phys. Chem. 101, 371-390 (1976)

13. Hogben, H. J., Biskup, T. \& Hore, P. J. Entanglement and sources of magnetic anisotropy in radical pair-based avian magnetoreceptors. Phys. Rev. Lett. 109, 220501 (2012).

14. Morgan, K. \& Pethig, R. Increase in dc dark conductivity of anthracene in a magnetic field. Nature 213, 900 (1967).

15. Frankevich, E. L., Sokolik, I. A., Kadyrov, D. I. \& Kobryanskii, V. M. Effect of a weak magnetic-field on the electrical-conductivity of polyacetylene films. JETP Lett. 36, 486-489 (1982).

16. Sheng, Y. et al. Hyperfine interaction and magnetoresistance in organic semiconductors. Phys. Rev. B 74, 045213 (2006).

17. Lee, S.-Y. et al. Tuning hyperfine fields in conjugated polymers for coherent organic spintronics. J. Am. Chem. Soc. 133, 2019-2021 (2011).

18. Nguyen, T. D., Gautam, B. R., Ehrenfreund, E. \& Vardeny, Z. V. Magnetoconductance response in unipolar and bipolar organic diodes at ultrasmall fields. Phys. Rev. Lett. 105, 166804 (2010).

19. Kersten, S. P., Schellekens, A. J., Koopmans, B. \& Bobbert, P. A. Magnetic-field dependence of the electroluminescence of organic light-emitting diodes: A competition between exciton formation and spin mixing. Phys. Rev. Lett. 106, 197402 (2011).

20. Lee, S.-Y. et al. Modulation frequency dependence of continuous-wave optically/electrically detected magnetic resonance. Phys. Rev. B 86, 115204 (2012).

21. Nguyen, T. D. et al. Isotope effect in spin response of pi-conjugated polymer films and devices. Nature Mater. 9, 345-352 (2010).

22. Frankevich, E. L., Pristupa, A. I. \& Kobryanskii, V. M. 1st observation of a magnetic-resonance change in the resistance of an organic semiconductor (weakly doped polyacetylene). JETP Lett. 40, 733-735 (1984).

23. Wohlgenannt, M., Tandon, K., Mazumdar, S., Ramasesha, S. \& Vardeny, Z. V. Formation cross-sections of singlet and triplet excitons in pi-conjugated polymers. Nature 409, 494-497 (2001).

24. Woodward, J. R., Timmel, C. R., McLauchlan, K. A. \& Hore, P. J. Radio frequency magnetic field effects on electron-hole recombination. Phys. Rev. Lett. 87, 077602 (2001)
25. Henbest, K. B., Kukura, P., Rodgers, C. T., Hore, P. J. \& Timmel, C. R. Radio frequency magnetic field effects on a radical recombination reaction: A diagnostic test for the radical pair mechanism. J. Am. Chem. Soc. 126, 8102-8103 (2004)

26. Wedge, C. J. et al. Spin-locking in low-frequency reaction yield detected magnetic resonance. Phys. Chem. Chem. Phys. 15, 16043-16053 (2013).

27. McCamey, D. R. et al. Hyperfine-field-mediated spin beating in electrostatically bound charge carrier pairs. Phys. Rev. Lett. 104, 017601 (2010)

28. Boehme, C. \& Lips, K. Theory of time-domain measurement of spin-dependent recombination with pulsed electrically detected magnetic resonance. Phys. Rev. B 68, 245105 (2003).

29. Mahato, R. N. et al. Ultrahigh magnetoresistance at room temperature in molecular wires. Science 341, 257-260 (2013).

30. Petta, J. R. et al. Coherent manipulation of coupled electron spins in semiconductor quantum dots. Science 309, 2180-2184 (2005).

\section{Acknowledgements}

This work was supported by the US Department of Energy, Office of Basic Energy Sciences, Division of Materials Sciences and Engineering under Award \#DESC0000909. The authors are indebted to M. Raikh and R. Roundy, whose theoretical work on a.c.-driven magnetoresistance ${ }^{3}$ inspired this study, for many insightful discussions. We thank J. Yu for preparing the deuterated MEH-PPV and S. Waters for help with graphic design.

\section{Author contributions}

D.P.W. and G.J. performed the experiments and data analysis. M.K. and D.P.W. prepared devices. G.J., H.M. and M.E.L. developed and implemented the experimental set-up. G.J. performed the calibration of the RF field amplitude $B_{1}$. D.P.W. and C.B. conceived the concept of these experiments. J.M.L. contributed to the discussion and guidance of the project. D.P.W., J.M.L., P.L.B. and C.B. contributed to manuscript preparation. P.L.B. supplied the deuterated MEH-PPV. C.B. and J.M.L. oversaw the project.

\section{Additional information}

Supplementary information is available in the online version of the paper. Reprints and permissions information is available online at www.nature.com/reprints. Correspondence and requests for materials should be addressed to J.M.L. or C.B.

\section{Competing financial interests}

The authors declare no competing financial interests. 


\section{Methods}

The MEH-PPV devices used in this study were fabricated on commercially acquired (SPI Supplies) indium tin oxide (ITO)-coated $700-\mu \mathrm{m}$-thick glass. The ITO was etched (Oxford Plasmalab80) to give an active area of $2 \times 3 \mathrm{~mm}^{2}$ contacted by a narrow strip of ITO. The lateral conductivity of the ITO strip was improved by depositing a 125-nm-thick layer of $\mathrm{Al}$ (Denton Sputterer) on top of the ITO strip, outside of the active OLED area, as described previousl $\mathrm{y}^{27}$. A 50-nm layer of PEDOT:PSS (Clevios) was spin-coated under ambient conditions followed by a $20 \mathrm{~min}$ thermal anneal at $120^{\circ} \mathrm{C}$ on a hotplate. Hydrogenated or deuterated ${ }^{17}$ $\mathrm{MEH}-\mathrm{PPV}$ layers were spin-coated from toluene solutions followed by thermal annealing for $10 \mathrm{~min}$ at $100^{\circ} \mathrm{C}$ on a hotplate in a nitrogen glovebox. The devices were completed by thermal evaporation of $5 \mathrm{~nm}$ of Ca capped with $150 \mathrm{~nm}$ of Al to form the cathode. Hydrogenated MEH-PPV was purchased from American Dye Source. For device verification, visual mechanical inspection and electrical characterization ( $I-V$ characteristics) were carried out followed by a second visual inspection under bias to confirm electroluminescence. $I-V$ characteristics of the two materials were found to be very similar, within standard sample-to-sample variations. The devices were encapsulated (using Araldite $2011 \mathrm{~A} / \mathrm{B}$ ) and then transferred from the glovebox to the experimental set-up. The experimental set-up (sketched in Fig. 1a) was developed and built in-house. The sample was mounted in air and connected using a printed circuit board located at the tip of a sample rod that could fit into the centre of the RF coil, which itself was located at the centre of two Helmholtz coils that provided the field $B_{0}$. The sample plane was perpendicular to the direction of $B_{0}$ and parallel to $B_{1}$. The control of $B_{0}$ was accomplished by a Kepco ATE 100-10M constant-current source. The continuous-wave RF field was generated by an Agilent MXG N5128A generator whose output was connected to an ENI 5100L RF (50 dB, $100 \mathrm{~W}, 1.5-400 \mathrm{MHz})$ amplifier. The RF pulses used for $B_{1}$ calibration were triggered by a Pulseblaster DDS-I-300 pulse generator. The device bias was provided by a $9 \mathrm{~V}$ battery connected to a potentiometer, so as to minimize electrical noise. The sample current was detected by an SRS570 amplifier (with a $10 \mathrm{~Hz}$ low-pass filter) linked to a NI PCI-6251 DAQ analog-digital converter for continuous-wave operation and to an AlazarTech ATS9462 digitizer for pulsed operation. MATLAB was used for data acquisition and processing. We note that the relative amplitude of the measured device current changes decreases with increasing sample current (and hence bias) as non-spin-dependent parallel (leakage) current becomes more prevalent. The results presented here were therefore recorded at low biases (just above OLED turn-on), where the ratio between spin-dependent and spin-independent currents is maximal. The current measurements reported here are based on averages of multiple repeated measurements, as described in the Supplementary Information. 